\title{
EXPONENTIALLY SMALL ESTIMATES FOR KAM THEOREM NEAR AN ELLIPTIC EQUILIBRIUM POINT
}

\author{
AMADEU DELSHAMS \\ Departament de Matemàtica Aplicada I, Universitat Politècnica de Catalunya \\ Diagonal 647, E-08028 Barcelona \\ AND PERE GUTIÉRREZ \\ Departament de Matemàtica Aplicada II, Universitat Politècnica de Catalunya \\ Pau Gargallo 5, E-08028 Barcelona
}

\begin{abstract}
We give a precise statement of KAM theorem for a Hamiltonian system in a neighborhood of an elliptic equilibrium point. If the frequencies of the elliptic point satisfy a Diophantine condition, with exponent $\tau$, and a nondegeneracy condition is fulfilled, we show that in a neighborhood of radius $r$ the measure of the complement of the KAM tori is exponentially small in $(1 / r)^{1 /(\tau+1)}$. This result is obtained by putting the system in Birkhoff normal form up to an appropriate order, and the key point relies on giving accurate estimates for its terms.
\end{abstract}

\section{Introduction}

We consider a Hamiltonian, with $n$ degrees of freedom, having the origin as an elliptic equilibrium point. In suitable canonical coordinates, this system can be put in the form

$$
H(q, p)=\sum_{s \geq 2} H_{s}(q, p),
$$

where $H_{s}$ is a homogeneous polynomial of degree $s$ in $(q, p)$ for every $s \geq 2$, and

$$
H_{2}(q, p)=\frac{1}{2} \sum_{j=1}^{n} \lambda_{j}\left(q_{j}^{2}+p_{j}^{2}\right) .
$$

We are concerned with the existence of $n$-dimensional invariant tori in a neighborhood of the elliptic point. We first see that the system (1-2) is nearly-integrable by putting it in Birkhoff normal form up to an appropriate degree $K \geq 4$, provided the frequency vector $\lambda=\left(\lambda_{1}, \ldots, \lambda_{n}\right)$ is nonresonant up to order $K$. A quantitative version of Birkhoff theorem, implicitly contained in [4], allows us to obtain estimates for the normal form. Like in [9], we consider action-angle variables in a neighborhood of radius $r$. Assuming a suitable nondegeneracy condition, we apply the known KAM theorem and show that most trajectories in a neighborhood of radius $r$ lie in invariant tori: we get for the relative measure of their complement an estimate of the type $\mathcal{O}\left(r^{(K-3) / 2}\right)$.

We assume that $\lambda$ satisfies a Diophantine condition: with given $\tau>n-1$ and $\gamma>0$,

$$
|k \cdot \lambda| \geq \frac{\gamma}{|k|^{\tau}} \quad \forall k \in \mathbb{Z}^{n} \backslash\{0\},
$$


where we write $|k|=\sum_{j=1}^{n}\left|k_{j}\right|$. We say $\lambda$ to be $\tau, \gamma$-Diophantine. Our main contribution is to show that in this case the estimates for the Birkhoff normal form allow us to choose the degree $K$ as a function of $r$, giving rise to an exponentially small estimate of the type

$$
\exp \left\{-\left(\frac{1}{r}\right)^{1 /(\tau+1)}\right\}
$$

for the measure of the complement of the invariant set. We remark that the estimates given in [4] do not allow to obtain the exponent $1 /(\tau+1)$, but a worse one. Nevertheless, we shall see that an improvement of that estimates leads to the announced exponent.

\section{Estimates for the Birkhoff normal form}

Given $K \geq 4$, assume that the frequency vector $\lambda$ is non resonant up to order $K: k \cdot \lambda \neq 0$ for $k \in \mathbb{Z}^{n}, 0<|k| \leq K$. The well-known Birkhoff theorem [1,7] states that, in some neighborhood of the origin, there exists a canonical transformation $\Psi^{(K)}$, near to the identity map, such that $\mathcal{H}^{(K)}=H \circ \Psi^{(K)}$ is in Birkhoff normal form up to degree $K$ :

$$
\mathcal{H}^{(K)}(q, p)=\lambda \cdot I+\mathcal{Z}^{(K)}(I)+\mathcal{R}^{(K)}(q, p)=h^{(K)}(I)+\mathcal{R}^{(K)}(q, p),
$$

with

$$
\mathcal{Z}^{(K)}(I)=\sum_{\substack{4 \leq s \leq K \\ s \text { even }}} \mathcal{Z}_{s}(I), \quad \mathcal{R}^{(K)}(q, p)=\sum_{s \geq K+1} \mathcal{R}_{s}^{(K)}(q, p),
$$

where every $\mathcal{Z}_{s}(I)$ (uniquely determined) is a homogeneous polynomial of degree $s / 2$ in the action variables

$$
I_{j}=\frac{1}{2}\left(q_{j}^{2}+p_{j}^{2}\right), \quad j=1, \ldots, n,
$$

and every $\mathcal{R}_{s}^{(K)}(q, p)$ is a homogeneous polynomial of degree $s$ in $(q, p)$. Since $h^{(K)}(I)$ is integrable, and in a neighborhood of radius $r$ we have $\mathcal{R}^{(K)}=\mathcal{O}\left(r^{K+1}\right)$, it turns out that $\mathcal{H}^{(K)}$ is a nearly-integrable Hamiltonian near the origin. However, to apply KAM theorem to $\mathcal{H}^{(K)}$ we need quantitative estimates for its terms.

As in [4], we introduce the linear change to complex canonical coordinates

$$
x_{j}=\frac{1}{\sqrt{2}}\left(q_{j}-i p_{j}\right), \quad y_{j}=-\frac{i}{\sqrt{2}}\left(q_{j}+i p_{j}\right), \quad j=1, \ldots, n .
$$

Note that $q, p$ are real if $\bar{y}=i x$. We define $|(x, y)|:=\max _{j=1, \ldots, n} \sqrt{\left|x_{j}\right|^{2}+\left|y_{j}\right|^{2}}$. Given $r>0$, the real and complex polydisks of radius $r$ centered at the origin will be denoted $\mathcal{B}_{r}$ and $\widehat{\mathcal{B}}_{r}$, respectively.

For a given homogeneous polynomial $f_{s}(x, y)=\sum_{|l+m|=s} f_{l, m} x^{l} y^{m}$, we define the norm

$$
\left\|f_{s}\right\|:=\sum_{|l+m|=s}\left|f_{l, m}\right|
$$

(we use the notation $x^{l}=x_{1}^{l_{1}} \cdots x_{n}^{l_{n}}, y^{m}=y_{1}^{m_{1}} \cdots y_{n}^{m_{n}}$ ). 
Proposition 1 Let $H(x, y)=\sum_{s>2} H_{s}$ be a real Hamiltonian with $H_{2}=\lambda \cdot I$, and assume that $\left\|H_{s}\right\| \leq c^{s-2} d$ for $s \geq 3$. Given $K \geq 4$, assume that

$$
|k \cdot \lambda| \geq \alpha_{K} \quad \forall k \in \mathbb{Z}^{n}, 0<|k| \leq K,
$$

with $0<\alpha_{K} \leq 1$. Then, there exists a real canonical transformation $\Psi^{(K)}$, near to the identity map, such that $\mathcal{H}^{(K)}=H \circ \Psi^{(K)}$ is in the Birkhoff normal form (4-5) up to degree $K$. With some constants $c_{1}, c_{2}$, one has:

a) $\left\|\mathcal{Z}_{s}\right\| \leq \frac{c_{2} c_{1}^{s-2}(s-2) \text { ! }}{\alpha_{3} \cdots \alpha_{s-1}}$ for $4 \leq s \leq K$ and s even.

b) $\left\|\mathcal{R}_{s}^{(K)}\right\| \leq \frac{c_{2} c_{1}^{s-2}(K-3) !(K-2)^{s-K+1}}{\alpha_{3} \cdots \alpha_{K-1} \alpha_{K}^{s-K+1}}$ for $s \geq K+1$.

c) The transformation $\Psi^{(K)}$ is analytic on $\widehat{\mathcal{B}}_{r_{K}^{*}}$, where we define $r_{K}^{*}:=\frac{\alpha_{K}}{c_{1} K}$.

These estimates rely on the results obtained in [4], although a direct application would give worse estimates, with $\alpha_{K}^{s-3}$ instead of $\alpha_{3} \cdots \alpha_{s-1}$ in the denominators. The improvement comes from the fact that, in the construction of the normal form, the only small divisors which appear up to the obtainment of $\mathcal{Z}_{s}$ correspond to the orders $3, \ldots, s-1$. This is crucial in order to get the right exponent in the estimates given in the last section.

\section{Applying KAM theorem}

We first recall a usual statement of KAM theorem. Let us consider a nearly-integrable Hamiltonian written in action-angle variables

$$
\mathcal{H}(\phi, I)=h(I)+f(\phi, I)
$$

with $\phi \in \mathbb{T}^{n}$ and $I \in \mathcal{G} \subset \mathbb{R}^{n}$. To show that most of the trajectories of $\mathcal{H}$ lie in $n$-dimensional invariant tori, one usually imposes one of the following nondegeneracy conditions on the frequency map $\omega=\nabla h$ :

$$
\operatorname{det}\left(\frac{\partial \omega}{\partial I}(I)\right) \neq 0 \quad \text { or } \quad \operatorname{det}\left(\begin{array}{cc}
\frac{\partial \omega}{\partial I}(I) & \omega(I) \\
\omega(I)^{\top} & 0
\end{array}\right) \neq 0
$$

for every $I \in \mathcal{G}$. We call these conditions Kolmogorov nondegeneracy and isoenergetic nondegeneracy, respectively.

We denote $\mathcal{V}_{\rho}(\mathcal{G})$ a complex neighborhood of radius $r$ around $\mathcal{G}$.

Theorem 2 (KAM theorem) Consider the Hamiltonian $\mathcal{H}=h(I)+f(\phi, I)$, analytic for $\phi \in \mathbb{T}^{n}$ and $I \in \mathcal{V}_{\rho}(\mathcal{G})$, with $f$ of size $\varepsilon$. Assume that $\omega=\nabla$ h is Kolmogorov or isoenergetically nondegenerate on $\mathcal{G}$. Let $\gamma>0$ given. For some constants $C_{1}, C_{2}, C_{3}$, if

$$
\varepsilon \leq C_{1} \gamma^{2}, \quad \gamma \leq C_{2} \rho,
$$

then there exists $\mathcal{I} \subset \mathbb{T}^{n} \times \mathcal{G}$ filled with $n$-dimensional invariant tori of $\mathcal{H}$, satisfying

$$
\text { mes }\left[\left(\mathbb{T}^{n} \times \mathcal{G}\right) \backslash \mathcal{I}\right] \leq C_{3}(\operatorname{diam} \mathcal{G})^{n-1} \gamma \text {. }
$$


For more detailed statements and proofs, see $[8,9,2,3,5]$. Given $\gamma$, it turns out that the invariant tori of $\mathcal{H}$ come from invariant tori of the unperturbed system $h$ with frequencies satisfying a Diophantine condition of the type (3), with a fixed $\tau$. Choosing $\gamma \sim \sqrt{\varepsilon}$, the measure of the complement in $(7)$ becomes $\mathcal{O}(\sqrt{\varepsilon})$.

Now, our aim is to apply KAM theorem to the Hamiltonian $\mathcal{H}^{(K)}=h^{(K)}+\mathcal{R}^{(K)}$ introduced in $(4-5)$. We put this Hamiltonian in action-angle variables by introducing the known canonical change

$$
q_{j}=\sqrt{2 I_{j}} \cdot \cos \phi_{j}, \quad p_{j}=\sqrt{2 I_{j}} \cdot \sin \phi_{j}, \quad j=1, \ldots, n .
$$

However, KAM theorem cannot be applied in a direct way because the change to actionangle variables is not analytic at the hyperplanes $I_{j}=0$. Like in [9], this fact forces us to remove a neighborhood of these hyperplanes. Thus, to obtain invariant tori in the neighborhood $\mathcal{B}_{r}$, we consider for the action variables the domain

$$
\mathcal{G}_{r, \rho}:=\left\{I \in \mathbb{R}^{n}: I \geq 2 \rho,|I|_{\infty} \leq \frac{r^{2}}{2}\right\}
$$

with $\rho>0$ (we use the notation $I \geq a$ to mean that $I_{j} \geq a$ for $j=1, \ldots, n$ ). With a suitable choice of $\rho$, this reduction of the domain does not affect essentially the measure estimates given in the next proposition.

To apply KAM theorem to $\mathcal{H}^{(K)}$, we also have to require that the frequency map $\omega^{(K)}=\nabla h^{(K)}$ is Kolmogorov or isoenergetically nondegenerate. In fact we only assume the nondegeneracy at the origin itself, since this suffices to ensure it in a small neighborhood. The condition we impose involves the vector $\lambda$ and the matrix

$$
A:=\frac{\partial^{2} \mathcal{Z}_{4}}{\partial I^{2}}
$$

Nevertheless, we point out that higher order conditions are also possible.

Proposition 3 In the same situation of proposition 1, assume also

$$
\operatorname{det} A \neq 0 \quad \text { or } \quad \operatorname{det}\left(\begin{array}{cc}
A & \lambda \\
\lambda^{\top} & 0
\end{array}\right) \neq 0
$$

Let $r_{K}^{*}$ defined as in part (c) of proposition 1. For some constants $c_{3}, c_{4}$, if

$$
0<r \leq c_{3} r_{K}^{*}
$$

then there exists $\mathcal{T}_{r}^{(K)} \subset \mathcal{B}_{r}$ filled with invariant tori of $\mathcal{H}^{(K)}$, satisfying

$$
\operatorname{mes}\left[\mathcal{B}_{r} \backslash \mathcal{T}_{r}^{(K)}\right] \leq c_{4}\left(\frac{7 r}{r_{K}^{*}}\right)^{(K-3) / 2} \cdot \operatorname{mes} \mathcal{B}_{r} .
$$

This result is obtained applying KAM theorem on the domain $\mathcal{V}_{\rho}\left(\mathcal{G}_{r, \rho}\right)$, with $\varepsilon \sim r^{K+1}$ and $\rho \sim r^{(K+1) / 2}$. In fact, this is a more elaborated version of a result given in [9], where a measure estimate like $(10)$ is obtained, also with the exponent $(K-3) / 2$. 


\section{The Diophantine case}

Finally, we assume that the frequency vector $\lambda$ satisfies the Diophantine condition (3) with given $\tau$ and $\gamma$. We then take $\alpha_{K}=\frac{\gamma}{K^{\tau}}$ in (6) and hence condition (9) is fulfilled if we choose $K \sim(\gamma / r)^{1 /(\tau+1)}$, leading to an exponentially small estimate.

Theorem 4 Let $H(x, y)=\sum_{s>2} H_{s}$ be a real Hamiltonian with $H_{2}=\lambda \cdot I$, and assume $\left\|H_{s}\right\| \leq c^{s-2} d$ for $s \geq 3$. Assume that $\lambda$ is $\tau, \gamma$-Diophantine, with $\tau>n-1$ and $\gamma>0$. Assume also one of the nondegeneracy conditions (8). For some constants $c_{5}, c_{6}, c_{7}$, if

$$
0<r \leq c_{5} \gamma
$$

then there exists $T_{r} \subset \mathcal{B}_{r}$ filled with invariant tori of $H$, satisfying

$$
\operatorname{mes}\left[\mathcal{B}_{r} \backslash T_{r}\right] \leq c_{6} \exp \left\{-\left(\frac{c_{7} \gamma}{r}\right)^{1 /(\tau+1)}\right\} \cdot \operatorname{mes} \mathcal{B}_{r} \text {. }
$$

A related result has been announced in [6] where, for a fixed KAM torus of a nearlyintegrable Hamiltonian, it is shown that in a neighborhood of radius $r$ there exist many invariant tori, and the measure of their complement is exponentially small in $1 / r$.

\section{Acknowledgements}

This work has been partially supported by the EC grant ERBCHRXCT940460. Research by Amadeu Delshams is also partially supported by the spanish grant DGICYT PB940215 and the catalan grant CIRIT GRQ93-1135, and research by Pere Gutiérrez is also partially supported by the U.P.C. grant PR9409.

\section{References}

1. G. D. Birkhoff (1927). "Dynamical systems". Am. Math. Soc. Colloq. Publ. 9. American Mathematical Society, New York.

2. H. W. Broer and G. B. Huitema (1991). "A proof of the isoenergetic KAM-theorem from the 'ordinary' one". J. Diff. Eq. 90, 52-60.

3. A. Delshams and P. Gutiérrez (1995). "Effective stability and KAM theory". Submitted to J. Diff. Eq.

4. A. Giorgilli, A. Delshams, E. Fontich, L. Galgani and C. Simó (1989). "Effective stability for a Hamiltonian system near an elliptic equilibrium point, with an application to the restricted three body problem". J. Diff. Eq. 77, 167-198.

5. P. Gutiérrez (1995). "Estabilitat efectiva i tors invariants de sistemes hamiltonians quasi-integrables". Doctoral thesis, Universitat de Barcelona.

6. A. Morbidelli and A. Giorgilli (1994). "Superexponential stability of KAM tori". To appear in J. Stat. Phys.

7. J. Moser (1968). "Lectures on Hamiltonian systems". Memoirs Am. Math. Soc. 81, 1-60.

8. A. I. Neishtadt (1982). "Estimates in the Kolmogorov theorem on conservation of conditionally periodic motions". J. Appl. Math. Mech. 45, 766-772.

9. J. Pöschel (1982). "Integrability of Hamiltonian systems on Cantor sets". Comm. Pure Appl. Math. 35, 653-696. 